\title{
About Majorana's Unpublished Manuscripts on Relativistic Quantum Theory for Particles of Any Spin
}

\author{
Laura Deleidi*, Marta Greselin
}

Dipartimento di Ingegneria e Scienze Applicate, Università Statale di Bergamo, Italy

Copyright (c)2015 Horizon Research Publishing All rights reserved.

\begin{abstract}
In this paper we analyze the formal and conceptual steps made by Ettore Majorana in a wide set of unpublished (handwritten) manuscripts (Quaderni, Fascicoli, Volumetti) written in later 20's and earlier 30's where, starting from the Dirac equation for spin- $\frac{1}{2}$ particles, he developed quantum relativistic wave equations for different (integer and half-integer) spins. In such a way Majorana obtained a Dirac-like equation for the photon and an infinite component quantum field theory for particles of any spin, thus anticipating the modern supersymmetry and string theories.
\end{abstract}

Keywords Majorana, Dirac Equation, Spinning Particles, Fierz-Pauli Theory, Supersymmetry

As is well known [1, 2], Majorana's scientific work has been only partially published. A large amount of studies, problems, notes, extemporaneous reminders and analytic calculations is still unpublished and represents a historical scientific corpus which is unique and incomparable of its kind [3]. The value of the handwritten manuscripts of the Sicilian physicist is not merely historiographical: some theories, formalisms and concepts - still to be investigated and developed - exhibit modern scientific features and can be applied to very different fields of theoretical physics.

The unpublished papers analyzed in this letter deal with the research of a formulation of a general theory for all elementary particles, which led Majorana first to the Dirac-like equation for photons and after, in 1932, to the infinite component field equation [4]

$$
\left(\Gamma_{\mu} \partial^{\mu}-m\right) \Psi=0
$$

a generalization to any spin of the ordinary Dirac equation, holding for spin- $\frac{1}{2}$ particles (hereafter $\hbar=c=1$ ). Before the infinite component field equation, Majorana wrote very general finite component field equations [5], describing particles with any spin, which can describe not elementary, but composite systems $[6,7,8]$. The finite component theory results to be equivalent to the standard theory of a generic spinning particle, mainly developed in Dirac's, Fierz's, and Pauli's [9] works. As is already evident in Eq. (1), Majorana's theoretical approach turns out to be very modern and has been recovered in the most recent relativistic quantum field theories.

The physicist Robert Oppenheimer, in an article published in 1931 [10], proposed a partially successful attempt to build a quantum theory of light. The Oppenheimer conjecture suggested to Majorana a Dirac-like equation for photons which, differently from Oppenheimer's equations, was not substantially different from quantum electrodynamics predictions. Majorana, rather than starting from the effective analogy between electron and photon, deduced the aforesaid equation directly from the Maxwell equations for the classical electromagnetic field. Thereby he overcame several analytical and conceptual difficulties which forced Oppenheimer to renounce the completion of his theory. Majorana's theory can be found in the later literature [11], in particular in Clifford Algebras [12] (Majorana's work, even if taken into account from the '70s [13], was ignored by the previously quoted authors who studied this problem, and is still little known). Let us remark that the formulation of a Dirac-like equation for the photon, for evident symmetry reasons, led to the Maxwelllike equation for the electron [14] and to extended Maxwell equations with Dirac magnetic monopoles [15].

The unpublished manuscripts, dated in the period 19281932, which we are referring to can be found in Fascicoli 8 and 9; in Quaderni: 1 pp. 14, 26, 37, 42, 48, 145, 150 / 2, pp. $81,86,101 / 1-7,113,137,141,150,184$ / 3, pp. 1, 2, 8, $11,20,25,36,71,144,160,170,180, \mathrm{~A} / 1-1, \mathrm{~A} / 4-3, \mathrm{C} / 1-1$, C/1-4, C/11-1, C/11-4 / 4 pp. 87, 143, 149, 154, 155, 158, 160, 171, 174 / 5 pp. 1, 5, 24, 131 / 6 pp. 6 / 7 pp. 161, 172, $180 / 12$ pp. 32,53 / 13 p. 3 / 15, pp. 10, 16, 22, $26 / 17$, pp. 45 , 69, 74, 83, 89, 146, 159, 176; in Volumetti: 3 cap. 18 p. 105 (written from 6/28/1929 to 4/23/1930) e 5 cap. 8 p. 36 .

Ettore Majorana starts from the 3-dimensional complex vector $\boldsymbol{F}=\boldsymbol{E}-i \boldsymbol{H}$, where vectors $\boldsymbol{E}$ and $\boldsymbol{H}$ indicate, as usual, the electric and magnetic field, respectively. The important physical rôle of $\boldsymbol{F}$ has been frequently recognized in the literature [16]. The picture of the (ordinary or generalized) classical electromagnetism in the algebraic framework of the so-called Spacetime Algebra [12], where the imaginary unit $i$ is replaced by the pseudoscalar $\gamma^{5}$ (which shows clearly the parity structure of $\boldsymbol{F}$ : scalar plus pseudoscalar), allows us to obtain a rather compact and elegant expression of the Maxwell equations [12, 16]. On the other hand [17], the only two relativistic independent invariants which can be realized by means of the electromagnetic tensor $F^{\mu \nu}$, i.e. 
$\frac{1}{2} F_{\mu \nu} F^{\mu \nu}=\boldsymbol{H}^{2}-\boldsymbol{E}^{2}$ and $\frac{1}{8} e_{i k l m} F^{i k} F^{l m}=\boldsymbol{E} \cdot \boldsymbol{H}$, are related to the real and to the imaginary part of the square of the vector $\boldsymbol{F}$, respectively. In place of the ordinary 4potential $A^{\mu}$, obeying a given gauge, Majorana introduces a vectorial photon wave function with 3 components defined as follows

$$
\boldsymbol{\psi} \equiv\left(\begin{array}{c}
E_{1}-i H_{1} \\
E_{2}-i H_{2} \\
E_{3}-i H_{3}
\end{array}\right)
$$

The probabilistic interpretation of the bilinear form $\boldsymbol{\psi}^{\dagger} \boldsymbol{\psi}$ has an immediate classical analogue, physically meaningful: in fact, it is equal to nothing else but $E^{2}+H^{2}$ which is, apart from a normalization constant, the energy density of the classical field. Actually, the electromagnetic energy density is proportional to the probabilistic density of photons (i.e., in field terms, to the expected number of photons per unit volume). Viceversa, the probabilistic meaning of the quantity $A_{\mu} A^{\mu}$ does not appear so direct and straightforward, and the rôle of the "wave function" $A^{\mu}$ in first quantization is not intuitive and conventional as well. The Maxwell equations can be rewritten in terms of $\psi$ as follows:

$$
\boldsymbol{\nabla} \cdot \boldsymbol{\psi}=\rho \quad i \boldsymbol{\nabla} \times \boldsymbol{\psi}=\boldsymbol{j}+\partial_{t} \boldsymbol{\psi} .
$$

In vacuum (free photons), with no charges and no currents, the first equation reduces to the so-called "transversality condition", usual in the standard wave mechanics for spin-1 particles

$$
\nabla \cdot \boldsymbol{\psi}=0
$$

while the second one can be suitably expanded

$$
\left\{\begin{array}{l}
i \partial_{t} \psi_{1}+i \partial_{y} \psi_{3}-i \partial_{z} \psi_{2}=0 \\
i \partial_{t} \psi_{2}+i \partial_{z} \psi_{1}-i \partial_{x} \psi_{3}=0 \\
i \partial_{t} \psi_{3}+i \partial_{x} \psi_{2}-i \partial_{y} \psi_{1}=0
\end{array}\right.
$$

At this point Majorana introduces the three hermitian matrices $\boldsymbol{\alpha}$

$$
\begin{gathered}
\alpha_{1} \equiv\left(\begin{array}{ccc}
0 & 0 & 0 \\
0 & 0 & i \\
0 & -i & 0
\end{array}\right) \alpha_{2} \equiv\left(\begin{array}{ccc}
0 & 0 & -i \\
0 & 0 & 0 \\
i & 0 & 0
\end{array}\right) \\
\alpha_{3} \equiv\left(\begin{array}{ccc}
0 & i & 0 \\
-i & 0 & 0 \\
0 & 0 & 0
\end{array}\right)
\end{gathered}
$$

which are a representation of the 3-dimensional group of the space rotations, and then satisfy the angular momentum algebra

$$
\left[\alpha_{i}, \alpha_{k}\right]=-i \varepsilon_{i k l} \alpha_{l}
$$

( $\varepsilon_{i k l}$ indicating the totally antisymmetric Levi-Civita tensor). By means of such matrices Majorana can actually write the wave equation for the photon in the form of a "DiracSchrödinger" equation for a massless (as is the photon) particle with spin $\frac{1}{2}$ :

$$
i \partial_{t} \psi=(\boldsymbol{\alpha} \cdot \widehat{\boldsymbol{p}}) \boldsymbol{\psi}
$$

Notice that from the previous equation it follows that the Hamiltonian for the free photon can be written as $\mathcal{H} \equiv \boldsymbol{\alpha} \cdot \widehat{\boldsymbol{p}}$. When $\psi$ is a plain wave, i.e. a momentum eigenfunction,

$$
\boldsymbol{\psi} \sim \mathrm{e}^{i(\boldsymbol{p} \cdot \boldsymbol{x}-\varepsilon t)}
$$

on applying Eq. (6) we just recover the usual photon momentum-energy dispersion relation $\varepsilon=|\boldsymbol{p}|$. The spin operator in the Hilbert space for such wave function is formally identical to the one of Dirac particles:

$$
\boldsymbol{\Sigma}=-i \boldsymbol{\alpha} \times \boldsymbol{\alpha}
$$

and has the expected eigenvalues $0, \pm 1$. Let us underline that the plane waves are at the same time eigenfunctions of momentum and helicity $\boldsymbol{s} \cdot \boldsymbol{p} /|\boldsymbol{p}|$.

Since the imaginary conjugate wave function $\psi^{\dagger}$ obeys the same wave equation

$$
i \partial_{t} \psi^{\dagger}=(\boldsymbol{\alpha} \cdot \widehat{\boldsymbol{p}}) \boldsymbol{\psi}^{\dagger}
$$

Majorana deduces that photons and antiphotons cannot be distinguished: e.g., they are really neutral particles.

The extension to non-free photons in the presence of charges or currents can be made directly from Eqs. (3), and leads to a generalized Dirac-like equation for massless particles because of the presence of $-i \boldsymbol{j}$, a sort of external field proportional to the electron current $\bar{\psi} \gamma \psi$.

Moreover, starting from the Dirac equation for spin- $\frac{1}{2}$ particles, Majorana tries to build Dirac-like equations for photons and particles with any spin. The standard theory for particles with spin larger than $\frac{1}{2}$ reduces, in substance, to the second-order Klein-Gordon equation with additional constraints and transversality conditions [9]. For integers and half-integers spins $>1$ it turns out to be impossible to formulate a variational principle by using only one (tensor or spinor) function, with rank corresponding to the given spin. To this end we need to introduce auxiliary tensor and spinor functions of lower rank. The Lagrangian is built in such a way that those auxiliary quantities cancel because of the field equations derived from the application of the variational principle.

In tensor notation (Fierz-Pauli theory) the wave function is an irreducible 4-tensor of rank $s$, i.e. a tensor symmetrical with respect to all indices and vanishing after contraction with respect to any couple of indices:

$$
\Psi_{. . \mu . \nu . .}=\Psi_{. . \nu . \mu . .} \quad \Psi_{. . \mu .}^{\mu . .}=0
$$

This tensor must satisfy the additional 4-dimensional transversality condition

$$
\partial^{\mu} \Psi_{. \mu . .}=0
$$

while any component obeys the Klein-Gordon equation

$$
\left(\square+m^{2}\right) \Psi_{\mu \nu \ldots}=0
$$

In the rest frame the previous equation implies the vanishing of all components of the 4-tensor with index 0 . As a matter of fact, the wave function in the center-of-mass frame (i.e., in the non-relativistic limit) reduces, as expected, to an irreducible rank- $s$ 3-dimensional tensor with $2 s+1$ independent components. Such equations can be replaced by a system of first-order equations holding for some suitable fields (whose choice is not unique, other equivalent groups of fields can be considered).

In spinor notation for any integer or half-integer spin we can write

$$
\left\{\begin{array}{c}
\left(\square+m^{2}\right) \psi_{\mu \nu \ldots}^{\dot{\alpha} \dot{\beta} \ldots}=0 \\
\varepsilon_{\dot{\alpha} \dot{\gamma}} \partial^{\dot{\gamma} \mu} \psi_{\mu \nu \ldots}^{\dot{\alpha} \dot{\beta} \ldots}=0
\end{array}\right.
$$


where $\psi$ is an irreducible spinor with $2 k$ undotted indices and $2 m-1$ dotted indices, symmetrical with respect to both indices. The spinor $\psi$ has $2 m(2 k+1)$ linearly independent components, which transform each other under the proper Lorentz group, according to the irreducible representation $D(k, m-1 / 2)$. The spin is either integer or half-integer depending on the total number of indices being even or odd.

Quite equivalently, Eqs. (12) can be replaced by the following first-order Dirac-like equations:

$$
\left\{\begin{array}{c}
\partial^{\dot{\alpha} \beta} \psi_{\beta \mu \nu . .}^{\dot{\gamma} \dot{\delta} \ldots}=m \phi_{\mu \nu \ldots}^{\dot{\alpha} \dot{\gamma} \dot{\delta} . .} \\
\partial_{\dot{\alpha} \beta} \phi_{\mu \nu \ldots}^{\dot{\alpha} \dot{\gamma} \dot{\delta} . .}=-m \psi_{\beta \mu \nu . .}^{\dot{\gamma} \dot{\delta} \ldots}
\end{array}\right.
$$

where $\psi$ and $\phi$ transform mutually each other under space inversion and then constitute a proper bispinor. Therefore in bispinorial notation the above equation system can be actually derived by the above seen Dirac-like equation

$$
\left(\Gamma_{\mu} \partial^{\mu}-m\right) \Psi=0
$$

where the matrices $\Gamma$ represent a suitable generalization of the ordinary Dirac algebra, with $\Psi$ indicating the aforesaid bispinor

$$
\Psi \equiv\left(\begin{array}{c}
\phi \\
\psi
\end{array}\right)
$$

Majorana promptly obtains the above Dirac-like equation in the presence of external fields by means of Euler-Lagrange equations, provided that the $\Gamma$ matrices realize representations (of suitable dimension) of the spacetime Clifford algebra $\mathbf{M}(1,3)$.

Both in Majorana's theory and in following papers [18, 19] the set of irreducible components of $\Psi$, analogously to what said for the tensor formulation, describes univocally a particle of a given spin only in the rest frame. As a matter of fact, in the general case with no supplementary conditions $\Psi$ can describe fields with variable spin: i.e., not elementary, but composite systems. Therefore Majorana's theory has two applications, depending on the interpretation of the wave function (or of the quantum field): arbitrary spin particles or composite systems. Majorana indeed tried to apply Eq. (1) to atomic and nuclear systems.

For what said above, the field $\Psi$ applies to bosons and fermions at the same time. It describes particles in a sense similar to the "supermultiplets", which are representations of fundamental supersymmetry groups found in present unification theories.

Majorana, on considering only the spacetime symmetries, searched for a finite-dimensional Hilbert space where all spins could be represented simultaneously as representations of the inhomogeneous Lorentz group. He found that this representation space exists, but it is infinite-dimensional, even if, with suitable boundary constraints, it could be "restricted" to a given spin. From a historical point of view, we could just say that the conceptual and formal Majorana's approach, fully developed, may represent the first proper example of particle supermultiplets, the last being "originated" by the mutual interaction among the particles themselves. The unpublished infinite component theory here analyzed was later independently re-discovered (with no knowledge of Majorana's work), by Gel'fand and Yaglom [20]. Then it was developed in the 60's and 70's in order to describe the Hydrogen atom or a generic hadron; to realize a Lagrangian quantum field theory for complex systems; to formulate an alternative theoretical approach to strong interactions based on the diffusion matrix and to develop dual resonance models which constitute the first steps in string theory; to define universe spinors non-locally coupled to the gravity.

\section{Acknowledgements}

The Authors gratefully acknowledge the scientific collaboration of G. Salesi. This work is partially supported by MIUR.

\section{REFERENCES}

[1] E.Recami: Il caso Majorana (Milan, 1987).

[2] E. Amaldi: La vita e l'opera di E. Majorana (Rome, 1966); "Ettore Majorana: Man and Scientist", in Strong and Weak Interactions, a cura di A. Zichichi (New York, 1966); E. Majorana, a cinquant'anni dalla sua scomparsa in Il Nuovo Saggiatore 1 (1988) pp.13-26.

[3] M. Baldo, R. Mignani e E. Recami: "Catalogo dei manoscritti scientifici inediti di E. Majorana" in E. Majorana - Lezioni all'Universiti $; \frac{1}{2}$ di Napoli pag. 175 (Naples, 1987)

[4] E. Majorana: Nuovo Cim. 9 (1932) 335.

[5] E. Majorana: Quaderno 3 pp.1-10.

[6] E. Majorana: Quaderno 1 pp.37-47; Quaderno 2, pp.86-96; Quaderno 4 pp.143-187.

[7] E.M. Corson: Introductions to tensors, spinors, and relativistic wave equations (Glasgow, 1953).

[8] L.D. Landau and E.M. Lifšits: Fisica Teorica, vol.IV: Teoria quantistica relativistica (Rome, 1978).

[9] P.A.M. Dirac: Proc. Roy. Soc. Lond. A155 (1936) 477; M. Fierz: Helv. Phys. Acta 12 (1939) 3; M. Fierz and W. Pauli: Proc. Roy. Soc. Lond. A173 (1939) 211.

[10] J.R. Oppenheimer: Phys. Rev. 38 (1931) 725.

[11] G. von Molière: Ann. Phys. (Leipzig) 6 (1949) 146; G.U. von Schubert: Ann. Phys. (Leipzg) 6 (1949) 163; W.L. Baade and H. Jehle: Rev. Mod. Phys. 23 (1953) 714; W.J. Archibald: Can. J. Phys. 33 (1955) 565; T. Ohmura: Progr. Theor. Phys. 16 (1956) 684; H.E. Moses: Suppl. Nuovo Cim. 7 (1958) 1; Phys. Rev. 113 (1959) 1670; O. Laporte and G.E. Uhlenbeck: Phys. Rev. 37 (1931) 1380; A.A. Campolattaro: Int. J. Theor. Phys. 19 (1980) 99; 19 (1980) 127; R.H. Good: Phys. Rev. 105 (1957) 1914.

[12] D. Hestenes: Space-time algebra (Gordon \& Breach; New York, 1966); New foundations for classical mechanics (Kluwer; Dordrecht, 1986); Found. Phys. 20 (1990) 1213; 23 (1993) 365; 15 (1985) 63; 12 (1981) 153; Am. J. Phys. 47 (1979) 399; 39 (1971) 1028; 39 (1971) 1013; J. Math. Phys. 14 (1973) 893; 16 (1975) 573; 16 (1975) 556; 8 (1979) 798; 8 (1967) 809; 8 (1967) 1046; D. Hestenes and G. Sobczyk: Clifford algebra to geometric calculus (Reidel; Dordrecht, 1984); R. Gurtler and D. Hestenes: J. Math. 
Phys. 16 (1975) 573; P. Lounesto: "Clifford algebras, relativity and quantum mechanics, in Gravitation: the spacetime structure (Proceedings of SILARG VIII), ed. by W.A. Rodrigues et al. (World Scientific; Singapore, 1994); I.M. Benn and R.W. Tucker: An introduction to spinors and geometry with applications in physics (Hilger; Bristol, 1987); A. Crumeyrolle: Orthogonal and symplectic Clifford algebras: Spinor structures (Kluwer; Dordrecht, 1990).

[13] R. Mignani, E. Recami e M. Baldo: Lett. Nuovo Cim. 11 (1974) 568; E. Giannetto: Lett. Nuovo Cim. 44 (1985) 140; 44 (1985) 145.

[14] S. Esposito and G. Salesi: Ann. Phys. (Berlin) 522, 456 (2010); A. Campolattaro: Int. J. Theor. Phys. 29 (1990) 141; W.A. Rodrigues Jr., J. Vaz Jr. and E. Recami: "Free Maxwell Equations, Dirac Equation, and Nondispersive de Broglie Wave-Packets" - in Courants, Amers, Écueils en Microphysique (G. and P. Lochak; 1994); J. Vaz and W.A. Rodrigues: Int. J. Theor. Phys. 6 (1993); "Is there any Relationship between Maxwell and Dirac Equations?" — preprint Imecc-Unicamp (Campinas, S.P., Brazil; 1994); C.W. Misner and J.A. Wheeler: Ann. Phys. 2 (1957) 525.

[15] A. Chubykalo and A. Espinoza: J. Adv. Phys. 8(1) (2015) 2010

[16] Ph. Gueret: Lectures at the Bari University (Bari, 1989); M. Faria-Rosa, E. Recami and W.A. Rodrigues: Phys. Lett. B173 (1986) 233; A. Maia, E. Recami, W.A Rodrigues and M.A.F. Rosa: J. Math. Phys. 31 (1990) 502; W.A. Rodrigues, E.Recami, A. Maia and M.A.F. Rosa: Phys. Lett. B220 (1989) 195.

[17] L.D. Landau and E.M. Lifšits: Fisica Teorica, vol.II: Teoria dei campi, pp.92-94 (Rome, 1978).

[18] Harish-Chandra: Phys. rev. 71 (1947) 793; Proc. Roy. Soc. Lond. A189 (1947) 372; A192 (1947) 195.

[19] H.J. Bhabha: Rev. Mod. Phys. 17 (1945) 200; Proc. Indian Acad. Sci. 21 (1945) 241.

[20] I.M. Gel'fand and A.M. Yaglom: Zh. Eksp. Teor. Fiz. 18 (1948) 703; 18 (1948) 1096; 18 (1948) 1105. 